\title{
Lambda-Nucleon and Sigma-Nucleon interactions from lattice QCD with physical masses
}

\author{
Hidekatsu Nemura ${ }^{*},{ }^{a}$ Sinya Aoki, ${ }^{a b}$ Takumi Doi, ${ }^{c}$ Shinya Gongyo, ${ }^{d}$ Tetsuo Hatsuda, ${ }^{c e}$ \\ Yoichi Ikeda, ${ }^{f}$ Takashi Inoue, ${ }^{g}$ Takumi Iritani, ${ }^{c}$ Noriyoshi Ishii, ${ }^{f}$ Takaya Miyamoto, ${ }^{b}$ \\ Keiko Murano $^{f}$ and Kenji Sasaki ${ }^{b}$ \\ ${ }^{a}$ Center for Computational Sciences, University of Tsukuba, Ibaraki, 305-8577, Japan \\ ${ }^{b}$ Center for Gravitational Physics, Yukawa Institute for Theoretical Physics, Kyoto University, \\ Kyoto, 606-8502, Japan \\ ${ }^{c}$ Theoretical Research Division, Nishina Center, RIKEN, Saitama, 351-0198, Japan \\ ${ }^{d}$ CNRS, Laboratoire de Mathématiques et Physique Théorique, Universitéde Tours, Tours, \\ France \\ e iTHES Research Group, RIKEN, Saitama, 351-0198, Japan \\ ${ }^{f}$ Research Center for Nuclear Physics (RCNP), Osaka University, Osaka 567-0047, Japan \\ ${ }^{g}$ Nihon University, College of Bioresource Sciences, Kanagawa 252-0880, Japan \\ E-mail: nemura.hidekatsu.gbeu.tsukuba.ac.jp
}

\begin{abstract}
We present our recent study on baryon-baryon $(B B)$ interactions from lattice QCD with almost physical quark masses corresponding to $\left(m_{\pi}, m_{K}\right) \approx(146,525) \mathrm{MeV}$ and large volume $(L a)^{4}=$ $(96 a)^{4} \approx(8.1 \mathrm{fm})^{4}$. In order to perform a comprehensive study of $B B$ interactions based on lattice QCD calculation with almost physical masses and to make better use of such large scale computer resources, a large number of $B B$ interactions from $N N$ to $\Xi \Xi$ are calculated simultaneously. In this report, we focus on the strangeness $S=-1$ channels of the hyperon interactions by means of HAL QCD method. The coupled-channel HAL QCD method is briefly outlined. The snapshots of central and tensor potentials in ${ }^{1} S_{0}$ and ${ }^{3} S_{1}-{ }^{3} D_{1}$ channels are presented for $\Lambda N, \Sigma N$ (both the isospin $I=1 / 2,3 / 2$ ) and their coupled-channel systems.
\end{abstract}

34th annual International Symposium on Lattice Field Theory

24-30 July 2016

University of Southampton, UK

\footnotetext{
* Speaker.
} 


\section{Introduction}

Precise determination of the $\Lambda$-nucleon $(\Lambda N)$ and the $\Sigma$-nucleon $(\Sigma N)$ interactions provides a significant impact for understanding how the hypernuclear systems are bound. It has been pointed out that a $\Lambda N-\Sigma N$ coupled-channel interaction plays a vital role to have a hypernucleus being bounded[1]. A recent experimental study shows a tendency to repulsive $\Sigma$-nucleus interaction and only a four-body $\Sigma$-hypernucleus $\left({ }_{\Sigma}^{4} \mathrm{He}\right)$ has been observed; those suggest a repulsive nature of the $\Sigma N$ interaction. Such understanding is useful to study properties of hyperonic matters inside the neutron stars, though a hyperonic equations of state (EOS) employed in such a study may contradict a recent observation of a massive neutron star heavier than $2 M_{\odot}[2,3]$.

In the recent years, a new lattice QCD approach to study the hadronic interactions has been proposed[4, 5]. In this approach, the interhadron potential is obtained by means of the lattice QCD measurement of the Nambu-Bethe-Salpeter (NBS) wave function. The observables such as the phase shifts and the binding energies are calculated by using the resultant potential[6]. This approach has been further extended and applied to various problems. See Refs.[7, 8] and references therein for the state-of-the-art outcomes. In addition, a large scale lattice QCD calculation is now in progress [9] to study the baryon interactions from $N N$ to $\Xi \Xi$ by measuring the NBS wave functions for 52 channels from the $2+1$ flavor lattice QCD.

The purpose of this report is to present our recent calculations of the $\Lambda N$ potentials as well as the $\Sigma N$ (both the isospin $I=1 / 2,3 / 2$ ) potentials using full QCD gauge configurations. Several earlier results had already been reported at LATTICE 2008, LATTICE 2009 and LATTICE 2011[10] with heavier quark masses and smaller lattice volumes. This report shows the latest results of those studies, based on recent works reported at LATTICE 2013[11, 12]; $\Lambda N-\Lambda N, \Lambda N-\Sigma N$, and $\Sigma N-\Sigma N$ (both $I=1 / 2$ and $3 / 2$ ) potentials are studied at almost physical quark masses corresponding to $\left(m_{\pi}, m_{K}\right) \approx(146,525) \mathrm{MeV}$ and large volume $(L a)^{4}=(96 a)^{4} \approx(8.1 \mathrm{fm})^{4}$.

\section{Outline of the HAL QCD method}

In order to study the nuclear force using the HAL QCD approach, we first define the equal time NBS wave function in particle channel $\lambda=\left\{B_{1}, B_{2}\right\}$ with Euclidean time $t[4,5]$

$$
\phi_{\lambda E}(\vec{r}) \mathrm{e}^{-E t}=\sum_{\vec{X}}\left\langle 0\left|B_{1, \alpha}(\vec{X}+\vec{r}, t) B_{2, \beta}(\vec{X}, t)\right| B=2, E, S, I\right\rangle,
$$

where $B_{1, \alpha}(x)\left(B_{2, \beta}(x)\right)$ denotes the local interpolating field of baryon $B_{1}\left(B_{2}\right)$ with mass $m_{B_{1}}\left(m_{B_{2}}\right)$, and $E=\sqrt{k_{\lambda}^{2}+m_{B_{1}}^{2}}+\sqrt{k_{\lambda}^{2}+m_{B_{2}}^{2}}$ is the total energy in the centre of mass system of a baryon number $B=2$, strangeness $S$, and isospin $I$ state. For $B_{1, \alpha}(x)$ and $B_{2, \beta}(x)$, we employ the local interpolating field of octet baryons given by

$$
\begin{aligned}
& p=\varepsilon_{a b c}\left(u_{a} C \gamma_{5} d_{b}\right) u_{c}, n=-\varepsilon_{a b c}\left(u_{a} C \gamma_{5} d_{b}\right) d_{c}, \Sigma^{+}=-\varepsilon_{a b c}\left(u_{a} C \gamma_{5} s_{b}\right) u_{c}, \Sigma^{-}=-\varepsilon_{a b c}\left(d_{a} C \gamma_{5} s_{b}\right) d_{c} \text {, } \\
& \Sigma^{0}=\frac{1}{\sqrt{2}}\left(X_{u}-X_{d}\right), \quad \Lambda=\frac{1}{\sqrt{6}}\left(X_{u}+X_{d}-2 X_{s}\right), \Xi^{0}=\varepsilon_{a b c}\left(u_{a} C \gamma_{5} s_{b}\right) s_{c}, \quad \Xi^{-}=-\varepsilon_{a b c}\left(d_{a} C \gamma_{5} s_{b}\right) s_{c}, \\
& \text { where } \quad X_{u}=\varepsilon_{a b c}\left(d_{a} C \gamma_{5} s_{b}\right) u_{c}, X_{d}=\varepsilon_{a b c}\left(s_{a} C \gamma_{5} u_{b}\right) d_{c}, \quad X_{s}=\varepsilon_{a b c}\left(u_{a} C \gamma_{5} d_{b}\right) s_{c} \text {. }
\end{aligned}
$$

For simplicity, we have suppressed the explicit spinor indices and spatial coordinates in Eq. (2.2) and the renormalization factors in Eq. (2.1). Based on a set of the NBS wave functions, we define 
a non-local potential $\left(\frac{\nabla^{2}}{2 \mu_{\lambda}}+\frac{k_{\lambda}^{2}}{2 \mu_{\lambda}}\right) \delta_{\lambda \lambda^{\prime}} \phi_{\lambda^{\prime} E}(\vec{r})=\int d^{3} r^{\prime} U_{\lambda \lambda^{\prime}}\left(\vec{r}, \vec{r}^{\prime}\right) \phi_{\lambda^{\prime} E}\left(\overrightarrow{r^{\prime}}\right)$ with the reduced mass $\mu_{\lambda}=m_{B_{1}} m_{B_{2}} /\left(m_{B_{1}}+m_{B_{2}}\right)$.

In lattice QCD calculations, we compute the four-point correlation function defined by[13]

$$
F_{\alpha \beta, J M}^{\left\langle B_{1} B_{2} \overline{B_{3} B_{4}}\right\rangle}\left(\vec{r}, t-t_{0}\right)=\sum_{\vec{X}}\left\langle 0\left|B_{1, \alpha}(\vec{X}+\vec{r}, t) B_{2, \beta}(\vec{X}, t) \overline{\mathscr{J}_{B_{3} B_{4}}^{(J, M)}\left(t_{0}\right)}\right| 0\right\rangle,
$$

where $\overline{\mathscr{J}_{B_{3} B_{4}}^{(J, M)}\left(t_{0}\right)}=\sum_{\alpha^{\prime} \beta^{\prime}} P_{\alpha^{\prime} \beta^{\prime}}^{(J, M)} \overline{B_{3, \alpha^{\prime}}\left(t_{0}\right) B_{4, \beta^{\prime}}\left(t_{0}\right)}$ is a source operator that creates $B_{3} B_{4}$ states with the total angular momentum $J, M$. The normalised four-point function can be expressed as

$$
\begin{aligned}
& R_{\alpha \beta, J M}^{\left\langle B_{1} B_{2} \overline{B_{3} B_{4}}\right\rangle}\left(\vec{r}, t-t_{0}\right)=\mathrm{e}^{\left(m_{B_{1}}+m_{B_{2}}\right)\left(t-t_{0}\right)} F_{\alpha \beta, J M}^{\left\langle B_{1} B_{2} \overline{B_{3} B_{4}}\right\rangle}\left(\vec{r}, t-t_{0}\right) \\
= & \sum_{n} A_{n} \sum_{\vec{X}}\left\langle 0\left|B_{1, \alpha}(\vec{X}+\vec{r}, 0) B_{2, \beta}(\vec{X}, 0)\right| E_{n}\right\rangle \mathrm{e}^{-\left(E_{n}-m_{B_{1}}-m_{B_{2}}\right)\left(t-t_{0}\right)}+O\left(\mathrm{e}^{-\left(E_{\mathrm{th}}-m_{B_{1}}-m_{B_{2}}\right)\left(t-t_{0}\right)}\right),(2.4
\end{aligned}
$$

where $E_{n}\left(\left|E_{n}\right\rangle\right)$ is the eigen-energy (eigen-state) of the six-quark system and $A_{n}=\sum_{\alpha^{\prime} \beta^{\prime}} P_{\alpha^{\prime} \beta^{\prime}}^{(J M M)}$ $\left\langle E_{n}\left|\bar{B}_{4, \beta^{\prime}} \bar{B}_{3, \alpha^{\prime}}\right| 0\right\rangle$. Hereafter, the spin and angular momentum subscripts are suppressed for $F$ and $R$ for simplicity. At moderately large $t-t_{0}$ where the inelastic contribution above the pion production $O\left(\mathrm{e}^{-\left(E_{\mathrm{th}}-m_{B_{1}}-m_{B_{2}}\right)\left(t-t_{0}\right)}\right)=O\left(\mathrm{e}^{-m_{\pi}\left(t-t_{0}\right)}\right)$ becomes negligible, we can construct the nonlocal potential $U$ through $\left(\frac{\nabla^{2}}{2 \mu_{\lambda}}+\frac{k_{\lambda}^{2}}{2 \mu_{\lambda}}\right) \delta_{\lambda \lambda^{\prime}} F_{\lambda^{\prime}}(\vec{r})=\int d^{3} r^{\prime} U_{\lambda \lambda^{\prime}}\left(\vec{r}, \vec{r}^{\prime}\right) F_{\lambda^{\prime}}\left(\overrightarrow{r^{\prime}}\right)$. In lattice QCD calculations in a finite box, it is practical to use the velocity (derivative) expansion, $U_{\lambda \lambda^{\prime}}\left(\vec{r}, \vec{r}^{\prime}\right)=$ $V_{\lambda \lambda^{\prime}}\left(\vec{r}, \vec{\nabla}_{r}\right) \delta^{3}\left(\vec{r}-\vec{r}^{\prime}\right)$. In the lowest few orders we have

$$
V\left(\vec{r}, \vec{\nabla}_{r}\right)=V^{(0)}(r)+V^{(\sigma)}(r) \vec{\sigma}_{1} \cdot \vec{\sigma}_{2}+V^{(T)}(r) S_{12}+V^{(L L S)}(r) \vec{L} \cdot\left(\vec{\sigma}_{1} \pm \vec{\sigma}_{2}\right)+O\left(\nabla^{2}\right),
$$

where $r=|\vec{r}|, \vec{\sigma}_{i}$ are the Pauli matrices acting on the spin space of the $i$-th baryon, $S_{12}=3(\vec{r}$. $\left.\vec{\sigma}_{1}\right)\left(\vec{r} \cdot \vec{\sigma}_{2}\right) / r^{2}-\vec{\sigma}_{1} \cdot \vec{\sigma}_{2}$ is the tensor operator, and $\vec{L}=\vec{r} \times(-i \vec{\nabla})$ is the angular momentum operator. The first three-terms constitute the leading order (LO) potential while the fourth term corresponds to the next-to-leading order (NLO) potential. By taking the non-relativistic approximation, $E_{n}-m_{B_{1}}-m_{B_{2}} \simeq \frac{k_{\lambda, n}^{2}}{2 \mu_{\lambda}}+O\left(k_{\lambda, n}^{4}\right)$, and neglecting the $V_{\mathrm{NLO}}$ and the higher order terms, we obtain $\left(\frac{\nabla^{2}}{2 \mu_{\lambda}}-\frac{\partial}{\partial t}\right) R_{\lambda \varepsilon}(\vec{r}, t) \simeq V_{\lambda \lambda^{\prime}}^{(\mathrm{LO})}(\vec{r}) \theta_{\lambda \lambda^{\prime}} R_{\lambda^{\prime} \varepsilon}(\vec{r}, t)$, with $\theta_{\lambda \lambda^{\prime}}=\mathrm{e}^{\left(m_{B_{1}}+m_{B_{2}}-m_{B_{1}^{\prime}}-m_{B_{2}^{\prime}}\right)\left(t-t_{0}\right)}$. Note that we have introduced the matrix form $R_{\lambda^{\prime} \varepsilon}=\left\{R_{\lambda^{\prime} \varepsilon_{0}}, R_{\lambda^{\prime} \varepsilon_{1}}\right\}$ with linearly independent NBS wave functions $R_{\lambda^{\prime} \varepsilon_{0}}$ and $R_{\lambda^{\prime} \varepsilon_{1}}$. For the spin singlet state, we extract the central potential as $V_{\lambda \lambda^{\prime}}^{(\text {Central })}(r ; J=0)=\left(\theta_{\lambda \lambda^{\prime}}\right)^{-1}\left(R^{-1}\right)_{\varepsilon^{\prime} \lambda^{\prime}}\left(\frac{\nabla^{2}}{2 \mu_{\lambda}}-\frac{\partial}{\partial t}\right) R_{\lambda \varepsilon^{\prime}}$. For the spin triplet state, the wave function is decomposed into the $S$ - and $D$-wave components as

$$
\left\{\begin{array}{l}
R\left(\vec{r} ;{ }^{3} S_{1}\right)=\mathscr{P} R(\vec{r} ; J=1) \equiv \frac{1}{24} \sum_{\mathscr{R} \in O} \mathscr{R} R(\vec{r} ; J=1), \\
R\left(\vec{r} ;{ }^{3} D_{1}\right)=\mathscr{Q} R(\vec{r} ; J=1) \equiv(1-\mathscr{P}) R(\vec{r} ; J=1) .
\end{array}\right.
$$

Therefore, the Schrödinger equation with the LO potentials for the spin triplet state becomes

$$
\left\{\begin{array}{l}
\mathscr{P} \\
\mathscr{Q}
\end{array}\right\} \times\left\{V_{\lambda \lambda^{\prime}}^{(0)}(r)+V_{\lambda \lambda^{\prime}}^{(\sigma)}(r)+V_{\lambda \lambda^{\prime}}^{(T)}(r) S_{12}\right\} \theta_{\lambda \lambda^{\prime}} R_{\lambda^{\prime} \varepsilon}\left(\vec{r}, t-t_{0}\right)=\left\{\begin{array}{l}
\mathscr{P} \\
\mathscr{Q}
\end{array}\right\} \times\left\{\frac{\nabla^{2}}{2 \mu_{\lambda}}-\frac{\partial}{\partial t}\right\} R_{\lambda \varepsilon}\left(\vec{r}, t-t_{0}\right),
$$




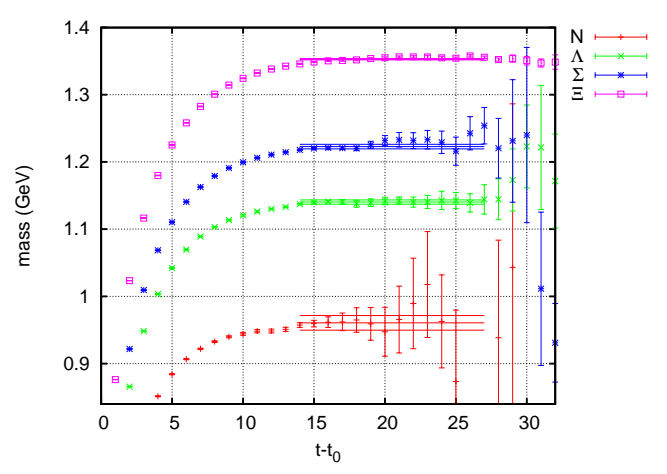

Figure 1: The effective mass of single baryon's correlation functions with utilising wall sources.

from which the central and tensor potentials, $V_{\lambda \lambda^{\prime}}^{(\text {Central })}(r ; J=0)=\left(V^{(0)}(r)-3 V^{(\sigma)}(r)\right)_{\lambda \lambda^{\prime}}$ for $J=0$, $V_{\lambda \lambda^{\prime}}^{(\text {Central })}(r ; J=1)=\left(V^{(0)}(r)+V^{(\sigma)}(r)\right)_{\lambda \lambda^{\prime}}$, and $V_{\lambda \lambda^{\prime}}^{(\text {Tensor })}(r)$ for $J=1$, can be determined ${ }^{1}$.

\section{Comprehensive lattice QCD calculation with almost physical quark masses}

$N_{f}=2+1$ gauge configurations at almost the physical quark masses are used; they are generated on $96^{4}$ lattice by employing the RG improved (Iwasaki) gauge action at $\beta=1.82$ with the nonperturbatively $O(a)$ improved Wilson quark (clover) action at $\left(\kappa_{u d}, \kappa_{s}\right)=(0.126117,0.124790)$ with $c_{s w}=1.11$ and the 6-APE stout smeared links with the smearing parameter $\rho=0.1$. Preliminary studies show that the physical volume is $(a L)^{4} \approx(8.1 \mathrm{fm})^{4}$ with the lattice spacing $a \approx 0.085 \mathrm{fm}$ and $\left(m_{\pi}, m_{K}\right) \approx(146,525) \mathrm{MeV}$. See Ref.[14] for details on the generation of the gauge configuration. The periodic (Dirichlet) boundary condition is used for spacial (temporal) directions; wall quark source is employed with Coulomb gauge fixing which is separated from the Dirichlet boundary by $\left|t_{D B C}-t_{0}\right|=48$. Forward and backward propagation in time are combined by using the charge conjugation and time reversal symmetries to double the statistics. Each gauge configuration is used four times by using the hypercubic $\mathrm{SO}(4, \mathbb{Z})$ symmetry of $96^{4}$ lattice. A large number of baryon-baryon potentials including the channels from $N N$ to $\Xi \Xi$ are studied by means of HAL QCD method[9]. See also Ref.[12] for the thoroughgoing consistency check in the numerical outputs and comparison at various occasions between the UCA[15] and the present algorithm[11]. In this report, 52 wall sources which is about a half (52/96) of possible statistics are used for the 207 gauge configurations at every 10 trajectories. Statistical data are averaged with the bin size 23. Jackknife method is used to estimate the statistical errors.

\section{Results}

\subsection{Effective masses from single baryons' correlation function}

As mentioned above, the potential is obtained at moderately large time slices where the inelastic contribution above the pion production is suppressed. In addition, the single baryon's correla-

\footnotetext{
${ }^{1}$ The potential is obtained from the NBS wave function at moderately large imaginary time; it would be $t-t_{0} \gg$ $1 / m_{\pi} \sim 1.4 \mathrm{fm}$. In addition, no single state saturation between the ground state and the excited states with respect to the relative motion, e.g., $t-t_{0} \gg(\Delta E)^{-1}=\left((2 \pi)^{2} /\left(2 \mu(L a)^{2}\right)\right)^{-1} \simeq 8.0 \mathrm{fm}$, is required for the HAL QCD method[13].
} 

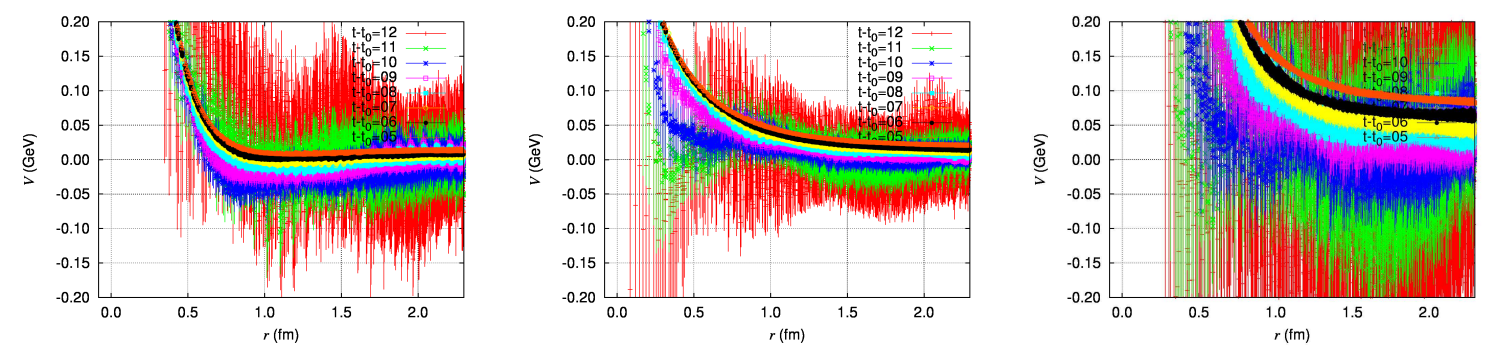

Figure 2: Left: $\Lambda N$ central potential in the ${ }^{1} S_{0}$ channel calculated with nearly physical point lattice QCD calculation on a volume $(96 a)^{4} \approx(8.1 \mathrm{fm})^{4}$ with the lattice spacing $a \approx 0.085 \mathrm{fm}$ and $\left(m_{\pi}, m_{K}\right) \approx(146,525) \mathrm{MeV}$. Centre: $\Lambda N-\Sigma N$ central potential in the ${ }^{1} S_{0}$ channel. Right: $\Sigma N$ central potential in the ${ }^{1} S_{0}$ channel.

tion functions, $\left(C_{B_{1}}\left(t-t_{0}\right) C_{B_{2}}\left(t-t_{0}\right)\right)^{-1}$, are used to obtain the normalised four-point correlation function instead of the simple exponential functional form $\mathrm{e}^{\left(m_{B_{1}}+m_{B_{2}}\right)\left(t-t_{0}\right)}$ in the actual numerical analysis. The statistical correlation between the numerator and the denominator in the normalised four-point correlation function maybe beneficial to reduce the statistical noise.

Fig. 1 shows the effective masses of the single baryon's correlation function. The plateau starts from the time slice around $t-t_{0} \approx 14$, which suggests that the potentials should be obtained at the time slices $t-t_{0} \geq 14$. However, statistics is still limited. In this report we present preliminary results at earlier time slices $\left(t-t_{0}=5-12\right)$ of our on-going work.

\subsection{Central potentials of $\Lambda N-\Sigma N$ in ${ }^{1} S_{0}$ channel}

Fig. 2 shows the $\Lambda N$ diagonal (left), $\Lambda N \rightarrow \Sigma N$ coupled-channel (centre), and $\Sigma N(I=1 / 2)$ diagonal (right) potentials in the ${ }^{1} S_{0}$ channel. In the flavor $S U(3)$ limit, these channels are expressed in terms of $\boldsymbol{8}_{s}$ and 27 representations, $|\Lambda N\rangle=\frac{1}{\sqrt{10}}\left(\left|\mathbf{8}_{s}\right\rangle+3|\mathbf{2 7}\rangle\right)$, and $|\Sigma N\rangle=\frac{1}{\sqrt{10}}\left(3\left|\mathbf{8}_{s}\right\rangle-|\mathbf{2 7}\rangle\right)$. Therefore the $\Lambda N$ diagonal potential is expected to be more or less similar to the $N N$ potential in the ${ }^{1} S_{0}$ channel. On the other hand, the $\Sigma N\left(I=1 / 2,{ }^{1} S_{0}\right)$ potential shows strong repulsive force which is consistent with the quark model's prediction.

\subsection{Central potentials of $\Lambda N-\Sigma N$ in ${ }^{3} S_{1}-{ }^{3} D_{1}$ channel}

Fig. 3 shows the $\Lambda N$ diagonal (left), $\Lambda N \rightarrow \Sigma N$ coupled-channel (centre), and $\Sigma N(I=1 / 2)$
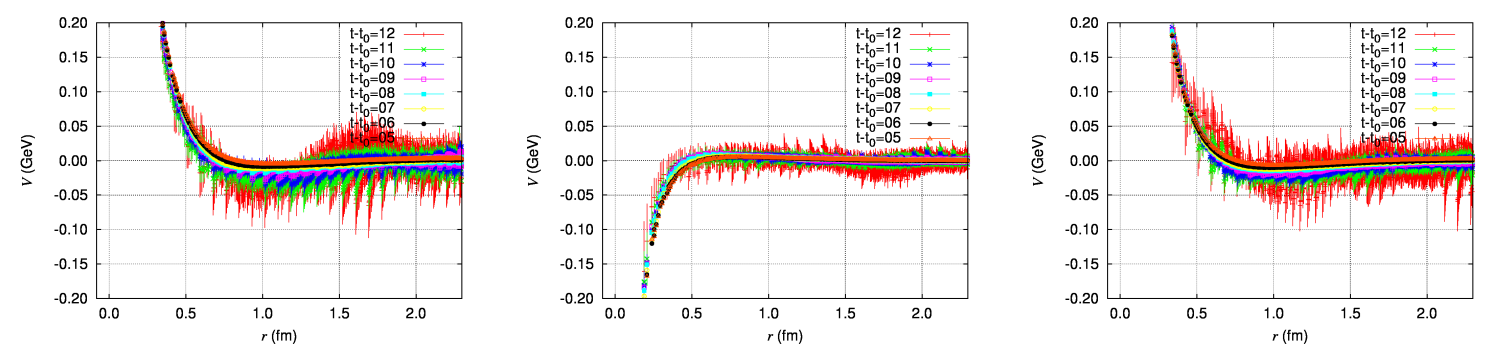

Figure 3: Left: $\Lambda N$ central potential in the ${ }^{3} S_{1}-{ }^{3} D_{1}$ channel calculated with nearly physical point lattice QCD calculation on a volume $(96 a)^{4} \approx(8.1 \mathrm{fm})^{4}$ with the lattice spacing $a \approx 0.085 \mathrm{fm}$ and $\left(m_{\pi}, m_{K}\right) \approx$ $(146,525) \mathrm{MeV}$. Centre: $\Lambda N-\Sigma N$ central potential in the ${ }^{3} S_{1}{ }^{3} D_{1}$ channel. Right: $\Sigma N$ central potential in the ${ }^{3} S_{1}-{ }^{3} D_{1}$ channel. 

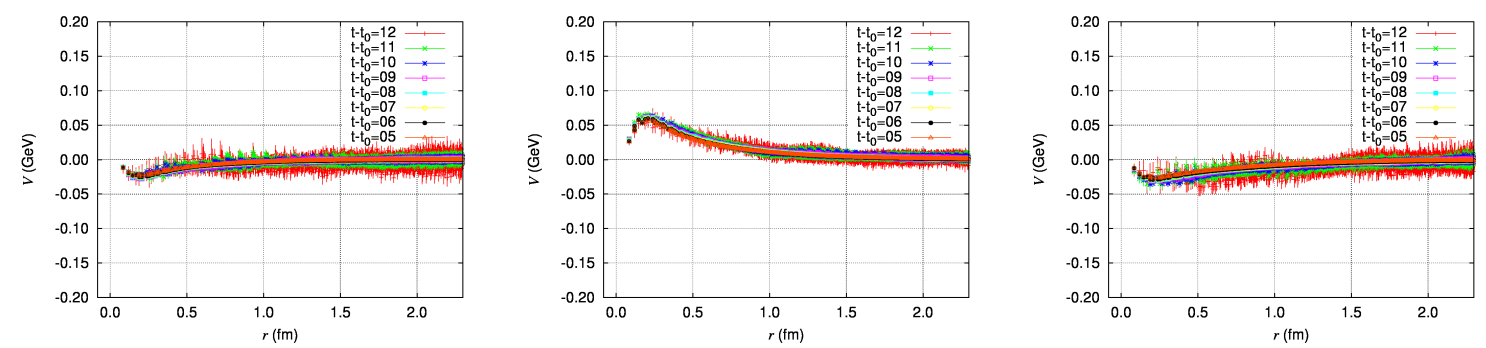

Figure 4: Left: $\Lambda N$ tensor potential in the ${ }^{3} S_{1}-{ }^{3} D_{1}$ channel calculated with nearly physical point lattice QCD calculation on a volume $(96 a)^{4} \approx(8.1 \mathrm{fm})^{4}$ with the lattice spacing $a \approx 0.085 \mathrm{fm}$ and $\left(m_{\pi}, m_{K}\right) \approx$ $(146,525) \mathrm{MeV}$. Centre: $\Lambda N-\Sigma N$ tensor potential. Right: $\Sigma N$ tensor potential.

diagonal (right) potentials in the ${ }^{3} S_{1}-{ }^{3} D_{1}$ channels. Relatively better signals are obtained in these states than in the ${ }^{1} S_{0}$ because of the three-times larger statistics in the spin triplet channel. In the both diagonal channels, repulsive core is found and weakly attractive dent seems to exist in the middle distance region. The off-diagonal (coupled channel) potential is seen at the short distance.

\subsection{Tensor potentials of $\Lambda N-\Sigma N$ in ${ }^{3} S_{1}-{ }^{3} D_{1}$ channel}

Fig. 4 shows the tensor potentials in the $\Lambda N$ (left), $\Lambda N \rightarrow \Sigma N$ (centre), and $\Sigma N(I=1 / 2)$ (right) potentials in the ${ }^{3} S_{1}-{ }^{3} D_{1}$ channel. Weak tensor potentials are seen in both diagonal channels. Regarding the study of light hypernuclear structure [1] the $\Lambda N-\Sigma N$ tensor potential is expected to play an important role to bind one or two $\Lambda$ ('s) and a light nucleus. The present result shows that the tensor potential has more or less sizable strength and it is weaker than the $N N$ tensor force.

\subsection{Two central $\left({ }^{1} S_{0},{ }^{3} S_{1}-{ }^{3} D_{1}\right)$ and a tensor $\left({ }^{3} S_{1}-{ }^{3} D_{1}\right)$ potentials of $\Sigma N(I=3 / 2)$ system}

Fig. 5 shows the two central potentials in the ${ }^{1} S_{0}$ (left) and ${ }^{3} S_{1}-{ }^{3} D_{1}$ (centre), and the tensor potential in the ${ }^{3} S_{1}-{ }^{3} D_{1}$ (right) channels of $\Sigma N(I=3 / 2)$ system, respectively. The ${ }^{1} S_{0} \Sigma N(I=$ $3 / 2$ ) channel is represented by pure 27 potential in the flavor $S U(3)$ limit which is same as the ${ }^{1} S_{0}$ $N N$ potential. The potential shows more or less similar to the $N N\left({ }^{1} S_{0}\right)$. On the other hand, the ${ }^{3} S_{1}-{ }^{3} D_{1}$ state is represented by pure 10 irreducible representation. The present results seems to suggest that the central potential in the ${ }^{3} S_{1}-{ }^{3} D_{1}$ is repulsive, which is consistent with the quark model's prediction. The tensor force is also obtained. The lattice QCD would be a promising approach to unveil the origin of repulsive nature of $\Sigma N$ interaction.
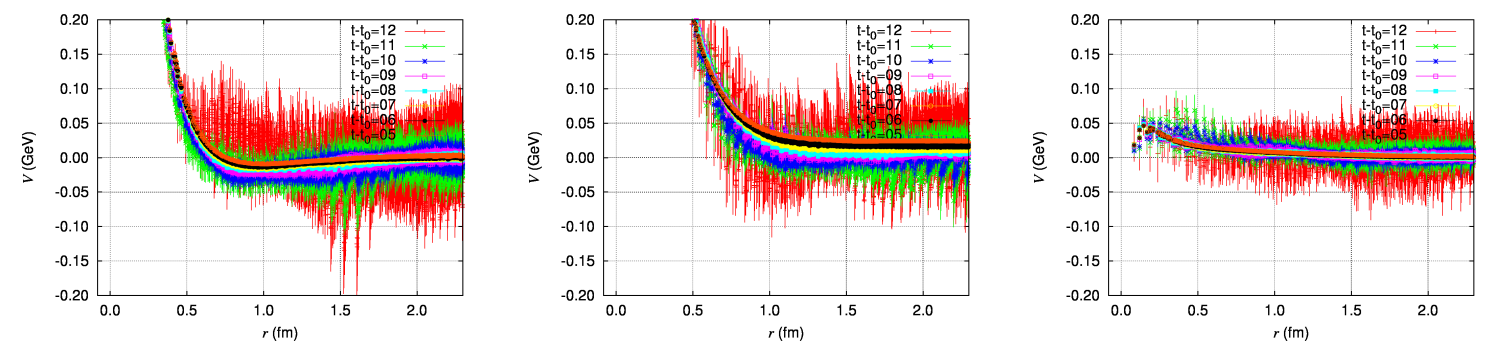

Figure 5: The $\Sigma N$ potentials of ${ }^{1} S_{0}$ central (left), ${ }^{3} S_{1}-{ }^{3} D_{1}$ central (centre), and ${ }^{3} S_{1}-{ }^{3} D_{1}$ tensor (right) in the $I=3 / 2$ channel. 


\section{Summary}

In this report, the preliminary snapshots of the $\Lambda N, \Sigma N$ and their coupled-channel potentials are presented. Both diagonal central potentials show the repulsive core in the short distance; the strengths are different from channel by channel. The ${ }^{1} S_{0} \Sigma N(I=1 / 2)$ and ${ }^{3} S_{1}-{ }^{3} D_{1} \Sigma N(I=3 / 2)$ show relatively stronger repulsive cores; it is interesting to see that the quark model predicts similar behaviour. In order to obtain more clear signals for both channels, several efforts should be devoted to improve the following points: (i) to increase statistics to go to larger time slices, (ii) to perform the analysis with taking into account the renormalization factors for the coupled channel potentials, (iii) to examine relativistic effects (i.e., higher differentials in time) for obtaining the potentials.

\section{Acknowledgments}

The lattice QCD calculations have been performed on the K computer at RIKEN, AICS ( hp120281, hp130023, hp140209, hp150223, hp150262, hp160211), HOKUSAI FX100 computer at RIKEN, Wako ( G15023, G16030) and HA-PACS at University of Tsukuba ( 14a-25, 15a-33, 14a-20, 15a-30). We thank ILDG/JLDG [16] which serves as an essential infrastructure in this study. This work is supported in part by MEXT Grant-in-Aid for Scientific Research (16K05340, 25105505, 15K17667, 25287046, 26400281, JP15K17667), and SPIRE (Strategic Program for Innovative Research) Field 5 project and "Priority issue on Post-K computer" (Elucidation of the Fundamental Laws and Evolution of the Universe). We thank all collaborators in this project.

\section{References}

[1] H. Nemura, Y. Akaishi and Y. Suzuki, Phys. Rev. Lett. 89 (2002) 142504 [arXiv:nucl-th/0203013].

[2] P. B. Demorest, et al., Nature 467, 1081 (2010).

[3] J. Antoniadis et al., Science 340, 6131 (2013) [arXiv:1304.6875 [astro-ph.HE]].

[4] N. Ishii, S. Aoki, T. Hatsuda, Phys. Rev. Lett. 99 (2007) 022001.

[5] S. Aoki, T. Hatsuda and N. Ishii, Prog. Theor. Phys. 123 (2010) 89.

[6] S. Aoki et al. [HAL QCD Collaboration], PTEP 2012 (2012) 01A105.

[7] K. Sasaki et al. [HAL QCD Collaboration], PTEP 2015, no. 11 (2015) $113 \mathrm{~B} 01$.

[8] Y. Ikeda et al. [HAL QCD Collaboration], Phys. Rev. Lett. 117, no. 24, 242001 (2016).

[9] T. Doi et al., in these proceedings; N. Ishii et al., in these proceedings; K. Sasaki et al., in these proceedings;

[10] H. Nemura [HAL QCD Collaboration], PoS LATTICE 2011, 167 (2011) [arXiv:1203.3320 [hep-lat]].

[11] H. Nemura [HAL QCD Collaboration], PoS LATTICE 2013, 426 (2014).

[12] H. Nemura, Comput. Phys. Commun. 207, 91 (2016) [arXiv:1510.00903 [hep-lat]].

[13] N. Ishii et al. [HAL QCD Collaboration], Phys. Lett. B 712, 437 (2012) [arXiv:1203.3642 [hep-lat]].

[14] K.-I. Ishikawa et al., PoS LATTICE 2015 (2015) 075 [arXiv:1511.09222 [hep-lat]].

[15] T. Doi and M. G. Endres, Comput. Phys. Commun. 184 (2013) 117.

[16] See http://www.lqcd.org/ildg and http://www.jldg.org 